\title{
Protein Folding with Python on Supercomputers
}

\author{
Jan H. Meinke ${ }^{\ddagger *}$
}

Abstract-Today's supercomputers have hundreds of thousands of compute cores and this number is likely to grow. Many of today's algorithms will have to be rethought to take advantage of such large systems. New algorithms must provide fine grained parallelism and excellent scalability. Python offers good support for numerical libraries and offers bindings to MPI that can be used to develop parallel algorithms for distributed memory machines.

PySMMP provides bindings to the protein simulation package SMMP. Combined with mpi4py, PySMMP can be used to perform parallel tempering simulations of small proteins on the supercomputers JUGENE and JuRoPA. In this paper, the performance of the Fortran implementation of parallel tempering in SMMP is compared with the Python implementation in PySMMP. Both codes use the same Fortran code for the calculation of the energy.

The performance of the implementations is comparable on both machines, but some challenges remain before the Python implementation can replace the Fortran implementation for all production runs.

Index Terms-parallel, MPI, biology, protein structure

\section{Introduction}

Many of the problems well known to high-performance computing (HPC) are becoming main stream. Processors add more and more cores, but the performance of a single core does not improve as drastically as it used to. Suddenly everybody has to deal with tens or hundreds of processes or threads. To take advantage of graphics hardware another factor of 100 in the number of threads is needed. Issues such as task management, load balancing, and race conditions are starting to become known to everybody who wants to write efficient programs for PCs. But things work the other way around, too. High-level programming languages such as Python that were not developed to get peak performance but to make good use of the developers time are becoming increasingly popular in HPC.

The Simulation Laboratory Biology at the Juelich Supercomputing Centre (JSC) uses Python to script workflows, implement new algorithms, perform data analysis and visualization, and to run simulations on the supercomputers JUGENE and JuRoPA. Often, we combine existing packages such as Biopython [BioPy], Modeller [MOD], matplotlib [PyLab], or PyQt [PyQt] with our own packages to tackle the scientific problems we are interested in. In this paper I will focus on using Python for protein folding studies on JuRoPA and JUGENE.

* Corresponding author: j.meinke@fz-juelich.de

¥Jülich Supercomputing Centre

Copyright $\odot 2010$ Jan H. Meinke. This is an open-access article distributed under the terms of the Creative Commons Attribution License, which permits unrestricted use, distribution, and reproduction in any medium, provided the original author and source are credited.

\section{Proteins}

Proteins bind oxygen and carbon dioxide, transport nutrients and waste products. They catalyze reactions, transfer information, and perform many other important functions. Proteins don't act in isolation. They are part of an interaction network that allows a cell to perform all the necessary operations of life. A very important feature of a protein is its shape. Only when it obtains its correct three dimensional structure does it provide the right interface for its reaction partners. In fact, changing the interface is a way to turn proteins on and off and regulate their activity.

Proteins are long chains of amino acids. The sequence of amino acids determines a protein's native shape. The sequence is encoded in the genome and assembled by the ribosome-itself a complex of RNA and proteins - amino acid by amino acid.

Proteins need anywhere from a few micro seconds to several minutes to obtain their native structure. This process is called protein folding. It occurs reliably in our body many times each second yet it is still poorly understood.

For some globular proteins it has been shown that they can unfold and refold in a test tube. At least for these proteins folding is driven purely by classical physical interactions. This is the basis for folding simulations using classical force fields.

The program package SMMP first released in 2001 [SMMP] implements several Monte Carlo algorithms that can be used to study protein folding. It uses a simplified model of a protein that keeps the bond angles and lengths fixed and only allows changes of the dihedral angles. To calculate the energy of a given conformation of a protein, SMMP also implements several energy functions, the so called force fields. A force field is defined by a functional form of the energy function and its parametrization.

In 2007, we released version 3 of SMMP [SMMP3]. With this version we provided Python bindings PySMMP that made the properties of the proteins, the algorithms, and the calculation of energy available from Python. In addition to the wrapper library created with f2py, we included three modules: universe, protein, and algorithms that make setting up a simulation and accessing the properties of a protein much more convenient. The wrapper modules were inspired by the Molecular Modeling Toolkit [MMTK], but implement a flat hierarchy. We did not, however, include the parallelized energy functions, which requires MPI to work.

For the work described in this paper, I decided to use mpi4py as MPI bindings for the Python code for its completeness and its integration with Scientific Python and Cython. An important feature of mpi4py is that it provides easy access to communicators in a way that can be passed to the Fortran subroutine called. 


\section{Compiling the Modules}

JUGENE is a 72-rack IBM Blue Gene/P (BG/P) system installed at JSC. Each rack consists of 1024 compute nodes. Each compute node has a 4-core PowerPC 450 processor running at $850 \mathrm{MHz}$ and 2 GB of memory for a total of 294912 cores and 147 TB of memory. The nodes are connected via a three dimensional torus network. Each node is linked to its six neighbors. In addition to the torus network, BG/P features a tree network that is used for global communication. The nodes are diskless. They forward IO requests to special IO nodes, which in turn talk to the GPFS file system. JUGENE's peak performance is about one petaflop and it reaches about 825 teraflops in the Linpack benchmark. This makes it Europe's fastest computer and the number 5 in the world [Top500]. While the slow clock rate makes the system very energy efficient (364 MFlops/W), it also makes code that scales well a must, since each individual core provides only about one third of the peak performance of an Intel Nehalem core and the performance gap is even larger in many applications. Production runs on JUGENE should use at least one rack.

Programs that run on JUGENE are usually cross-compiled for the compute nodes. The compute nodes run a proprietary 32-bit compute node kernel with reduced functionality whereas the login nodes use Power6 processors with a full 64-bit version of SUSE Linux Enterprise Server 10. Cross compiling can be tricky. It is important to set all the environment variables and paths correctly. First, we need to make sure to use the correct compiler

export BGPGNU=/bgsys/drivers/ppcfloor/gnu-linux export F90=\$BGPGNU/powerpc-bgp-linux/bin/gfortran

Then we need to use f2py with the correct Python interpreter, for example

export LD_LIBRARY_PATH=\$LD_LIBRARY_PATH: $\$ B G P G N U / 1$ ib \$BGPGNU/bin/python/bgsys/local/numpy/1.2.1/bin/f2py

Now, f2py produces libraries that can be loaded on the compute nodes.

\section{Launching Python on thousands of cores}

A first step for any Python program is to load the interpreter and the default modules. While this is usually not a problem if we start a few instances, it can become troublesome on a large system such as JUGENE.

Taking a look at the first two columns in Table 1 we see that already for a single rack, it takes more than 5 minutes to run a simple helloworld program using the default Python installation location. A C++ program for comparison takes only 5 s. Plotting the run time of the helloworld program, we quickly see that the time increases linearly with the number of MPI tasks at a rate of $0.1 \mathrm{~s}$ per task (Blue squares in Figure 1). Extrapolating this to all 294912 cores of JUGENE, it would take more than 8 hours to start the Python interpreter resulting in 25 lost rack days (70 CPU years with 4 cores per CPU) and almost 10 metric tons of $\mathrm{CO}_{2}$.

The linear behavior hints at serialization when the Python interpreter is loaded. As mentioned above, JUGENE's, compute nodes don't have their own disks. All IO is done via special IO nodes from a parallel file system and all nodes access the same Python image on the disk.

A similar behavior was discussed for the GPAW code in the mpi4py forum [PyOn10k]. GPAW [GPAW] uses its own Python MPI interface. Their work around was to use the ram disks of the IO nodes on Blue Gene/P.

\begin{tabular}{|c|c|c|c|}
\hline \# of Cores & Time $[\mathrm{s}]$ & Time $[\mathrm{s}]$ & Comments \\
\hline 1 & 5 & & \\
\hline 128 & 50 & 20 & A single node card \\
\hline 512 & 55 & & Midplane in SMP mode \\
\hline 1024 & 100 & & Only rank 0 writes \\
\hline 2048 & 376 & & $195 \mathrm{~s}$ if only rank 0 writes \\
\hline 4096 & 321 & 130 & $\begin{array}{l}1 \text { rack (smallest size for pro- } \\
\text { duction runs) }\end{array}$ \\
\hline 8192 & 803 & 246 & 2 racks \\
\hline 16384 & 1817 & 371 & $\begin{array}{l}4 \text { racks. For comparison, a } \\
\mathrm{C}++ \text { program takes } 25 \mathrm{~s} .\end{array}$ \\
\hline 20480 & & 389 & 5 racks \\
\hline 32768 & & 667 & 8 racks \\
\hline 65536 & & 927 & 16 racks \\
\hline 131071 & & 1788 & 32 rack \\
\hline
\end{tabular}

TABLE 1: Time measured for a simple MPI hello world program written using mpitpy on the Blue Gene/P JUGENE. The second column gives the times using the default location for Python on Blue Gene. The third column lists the times if Python is installed in the Work file system.

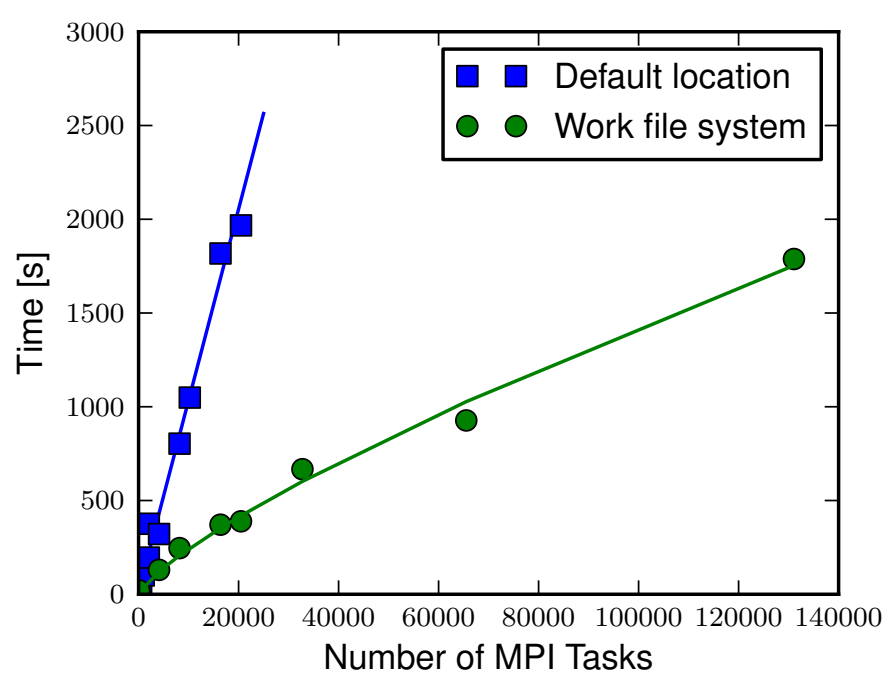

Fig. 1: Scaling of the startup time of the Python interpreter on JUGENE before and after optimization. Using the default location of the Python installation, the startup time increases linearly with the number of MPI tasks. Moving the Python installation to the faster Work file system reduces the scaling exponent from 1 to 0.77 .

Based on this data, we filed a service request with IBM. After some experimentation, IBM finally suggested to install Python on the Work file system. The Work file system is usually used as a skratch space for simulation data that is written during a run. Its block size of $2 \mathrm{MB}$ is optimized for large files and it reaches a bandwidth of $30 \mathrm{~GB} / \mathrm{s}$. Files written to the Work file system usually are deleted automatically after 90 days. In comparison the system and home file systems use a block size of $1 \mathrm{MB}$ and reach a bandwidth of $8 \mathrm{~GB} / \mathrm{s}$.

With Python installed on the Work file system, the scaling of the runtime of the helloworld program becomes sublinear with an exponent of about 0.77 (see column three in Table 1 and green disks in Figure 1). This make production runs of up to 32 racks 


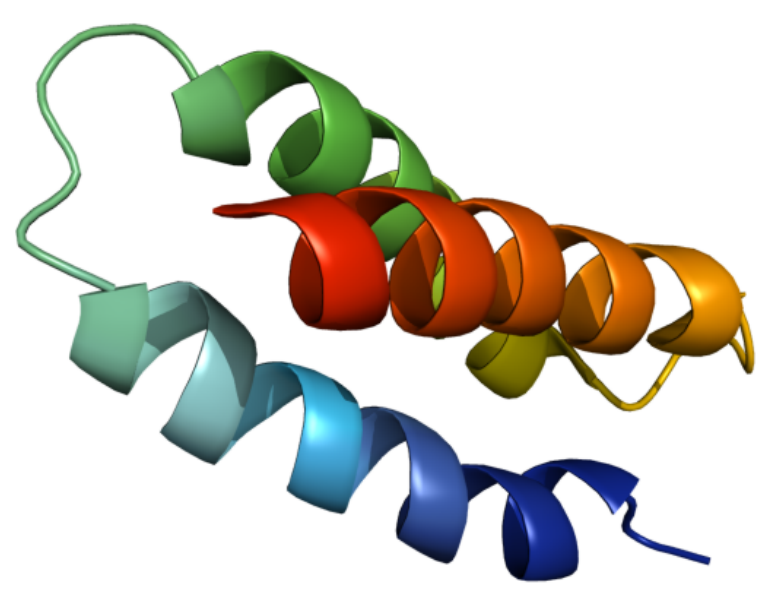

Fig. 2: Cartoon rendering of the three-helix bundle GS- $\alpha_{3} W$. The rendering was done with PyMOL [PyMOL].

(131071 cores) feasible. Extrapolating the data to 72 racks, it would now take less than an hour to start a run on the entire machine.

I also ran the same test on our second supercomputer, JuRoPA. JuRoPA is an Intel Nehalem cluster. Each of its 3288 nodes has two quad-core processors with $24 \mathrm{~GB}$ of memory for a total of 26304 cores and 79 TB of main memory. It has a peak performance of 308 teraflops and is currently number 14 in the Top 500 list with $90 \%$ efficiency in the Linpack benchmark [Top500]. It uses Infiniband in a fat tree topology for communication and a Lustre file system for storage. In contrast to JUGENE, each node has its own local disk, where Python is installed. While the time to start Python and load mpi4py.MPI still increases linearly with the number of nodes, the prefactor is only $0.005 \mathrm{~s}$ per process.

\section{Parallel energy calculation}

As mentioned above, the energy calculation for the ECEPP/3 force field and the associated implicit solvent term are parallelized. Before they can be used, however, the appropriate communicator needs to be defined. For most simulations, except parallel tempering (see Section Parallel tempering), the communicator is a copy of the default communicator that includes all processes. To start, such a simulation, we need to assign this communicator to smmp.paral.my_mpi_comm. This must be the appropriate Fortran reference, which we can get using mpi4py.MPI.COMM_WORLD.py2f(). With this setup, we can now compare the speed and the scaling of the energy function when called from Python and Fortran.

Scaling in parallel programs refers to the speedup when the program runs on $p$ processors compared to running it on one processor. If the run time with $p$ processors is given by $t(p)$ then the speedup $s$ is defined as $s(p)=t(1) / t(p)$ and the efficiency of the scaling is given by $e(p)=s(p) / p$. An efficiency of $50 \%$ is often considered acceptable.

As a benchmark system, I used the three-helix bundle GS$\alpha_{3} W$ (PDB code: 1LQ7) with 67 amino acids and 1110 atoms (see Figure 2).

On JuRoPA, I used f2py's default optimization options for the Intel compiler to create the bindings. The Fortran program was compiled with the -fast option, which activates most optimizations and includes interprocedural optimizations. For a single core, the

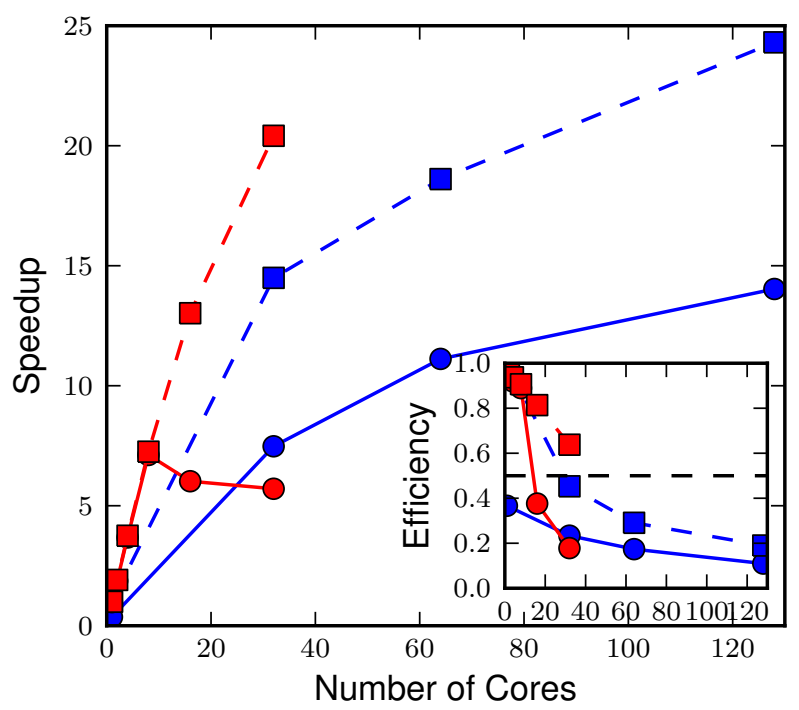

Fig. 3: Parallel scaling of the duration of the energy calculation for the three-helix bundle GS- $\alpha_{3} W$ on JuRoPA (red) and JUGENE (blue). The speedup is relative to the time needed by the Fortran program for the calculation of the energy on a single core. The square symbols represent SMMP, the disks PySMMP.

Fortran program is about $10 \%$ faster. The scaling on a single node is comparable, but it breaks down for PySMMP if more than one node is used (see Figure 3). This may be due to interactions between mpi4py and JuRoPA's MPI installation.

On JUGENE, the behavior is quite different. PySMMP was compiled with gfortran, SMMP with IBM's xlf compiler, which produces code that is almost three times faster on a single core. The shape of the scaling is comparable and saturates at about 128 cores.

\section{Parallel tempering}

Parallel tempering [PT], also known as replica exchange, is a method to sample a rough energy landscape more efficiently. Several copies of a system are simulated at different temperatures. In addition to regular Monte Carlo [MC] moves that change a configuration, we introduce a move that exchanges conformations of two different temperatures. The probability for such a move is $P_{\mathrm{PT}}=\exp (\Delta \beta \Delta E)$, where $\beta=1 / k_{B} T, T$ is the temperature and $k_{B}$ is the Boltzmann constant. With this exchange probability the statistics at each temperature remains correct, yet conformations can move to higher temperatures where it is easier to overcome large barriers. This allows for a more efficient sampling of the conformational space of a protein.

Parallel tempering is by its very nature a parallel algorithm. At each temperature, we perform a regular canonical MC simulation. After a number of updates $n_{u p}$, we attempt an exchange between temperatures. If we create our own MPI communicators, we can use two levels of parallelism. For each temperature $T_{i}$, we use a number of processors $p_{i}$ to calculate the energy in parallel. Usually, $p_{i}$ is the same for all temperatures, but this is not a requirement. Assuming that $p_{i}=p$, and using $n_{T}$ temperatures, we use a total of $p_{\mathrm{tot}}=n_{T} * p$ processors. For an average protein domain consisting of about 150 amino acids and 3000 atoms, $p=128$, and $n_{T}=64$ is a reasonable choice on a Blue Gene/P, for a total of $p_{\text {tot }}=8192$ - a good size for a production run. 


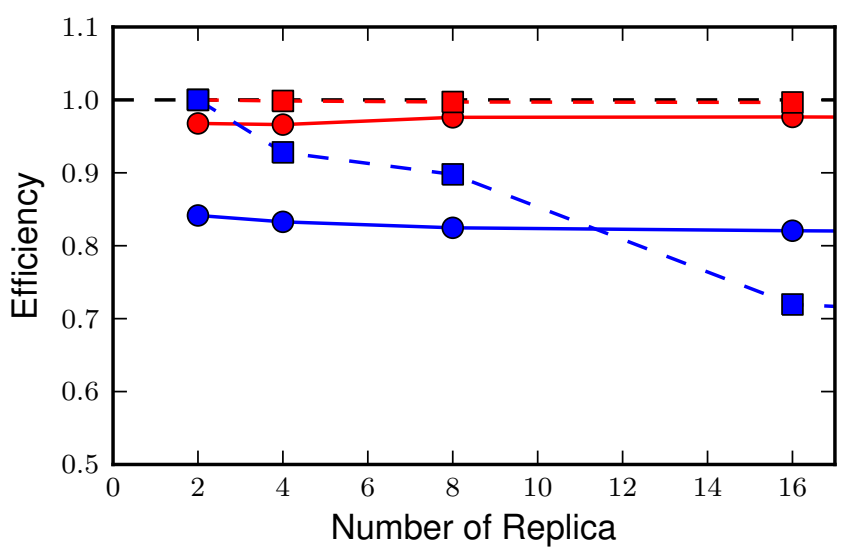

Fig. 4: Efficiency of the scaling of parallel tempering. Parallel tempering is an example for weak scaling. The problem size, i.e., the number of temperatures, increases proportional to the number of processors. Ideally, the time stays constant and the efficiency is one. For JuRoPA (red), both lines are nearly constant. The Python implementation (disks) of parallel tempering takes only about 5\% longer than the Fortran version (squares). On JUGENE (blue) each replica uses 128 cores for the energy calculation. The Python implementation takes about 20\% longer for 2 replica than the Fortran implementation but for 16 replica the difference is down to about $10 \%$.

Parallel tempering is implemented in Fortran as part of SMMP. The speed of the Fortran implementation is the reference, for the following investigation of my implementation of parallel tempering in Python. Parallel tempering and canonical Monte Carlo are implemented as classes in the algorithms module. The canonical Monte Carlo class optionally uses the Fortran implementation of the Metropolis step. For the following comparison, only the calculation of the energy of a conformation is done in Fortran.

For parallel tempering, the number of processes increases proportionally with the number of replicas. This kind of scaling is called weak scaling. Ideally, the time stays constant. Figure 4 shows the scaling of parallel tempering on JuRoPA and JUGENE with respect to the pure Fortran program. On JuRoPA, one node was used per replica. On JUGENE 128 cores were used per replica. The overhead of implementing the algorithm in Python is about $5 \%$ on JuRoPA and the scaling is comparable to the Fortran code. On JUGENE, the overhead of the Python implementation is about $20 \%$ for 2 replicas. But the scaling of PySMMP is better and for 16 replicas, the Python version takes only about $10 \%$ longer.

\section{Clustering}

In addition to scalar properties such as energy, volume, secondary structure content, and distance to the native structure, we can save the conformation, i.e., the coordinates of the structures, we have seen. We can create histograms that show us for each temperature, how often, we found structures that had a distance to the native conformation that fell into a certain range. A commonly used measure is the root-mean-square deviation (rmsd) of the current conformation to the native one. Rmsd measures the average change in position of all atoms compared to a reference structure. Unfortunately, rmsd is not a very good measure. For small rmsd values, two structures that have a similar rmsd to the native structure, will also be similar to each other, but for larger rmsd values this is not the case. To determine, the recurrence and therefore the statistical weight of structures that are very different from a given reference structure, we can use clustering algorithms. A cluster can be defined in many different ways. Three intuitive definitions are

- Elements belong to the same cluster if their distance to each other is less than a given distance $d_{\text {cluster }}$.

- Elements belong to the same cluster if they have more connections to each other than to other elements.

- Two clusters are distinct if the density of elements within the cluster is much higher than between clusters.

The first definition works well with rmsd as distance measure if we choose $d_{\text {cluster }}$ small enough and is an intuitive definition for clusters of structures, but it is computationally expensive. We usually have several tens of thousands of structures requiring billions of rmsd calculations to complete the distance matrix. We therefore started to look at alternatives. One alternative is to look for dense regions in high-dimensional spaces (the third definition). MAFIA [MAFIA] is a adaptive grid algorithm to determine such clusters. It looks for dense regions in increasingly higher dimension. A one-dimensional region is considered dense if the number of elements is larger than a threshold $n_{t}=\alpha \bar{n} w$, where $\alpha$ is a parameter, $\bar{n}$ is the average density of elements in that dimension, and $w$ is the width of the region. An n-dimensional region is considered dense if the number of elements it contains is larger than the threshold of each of its one-dimensional sub spaces For each dimension, MAFIA divides space into $n_{\text {bins }}$ uniform bins (see Figure 5). For each bin, it counts the number of elements in that bin creating a histogram. The next step is to reduce the number of bins by enveloping the histogram using $n_{\text {windows }}$ windows. The value of each window is the maximum of the bins it contains. To build an adaptive grid, neighboring windows are combined into larger cells if their values differ by less than a factor $\beta$. For each adaptive-grid cell, the threshold $n_{t}$ is calculated. The onedimensional dense cells are used to find two dimensional candidate dense units. The algorithm combines the dense units found to find increasingly higher-dimensional dense units. It takes advantage of the fact that all $n$-1-dimensional projections of an $n$-dimensional dense unit are also dense to quickly reduce the number of higherdimensional cells that need to be tested.

Since, we couldn't find an implementation of MAFIA, I implemented a Python version using NumPy and mpi4py. MAFIA combines task and data parallelism making it a good candidate for parallel compute clusters. The implementation consists of less than 380 lines of code, scales well, and can deal easily with tens of thousands of data points.

We are currently testing the usefulness of various ways to describe protein conformations as multi-dimensional vectors for clustering using PyMAFIA.

\section{Conclusions}

Today's supercomputers consist of tens to hundreds of thousands of cores and the number of cores is likely to grow. Using these large systems efficiently requires algorithms that provide a lot of parallelism. Python with mpi4py provides an avenue to implement and test these algorithms quickly and cleanly. The implementation of MAFIA shows that prototyping of a parallel program can be done efficiently in pure Python

On JuRoPA, the overhead of using Python instead of Fortran for the parallel tempering algorithm, is only about $3 \%$ if the energy 


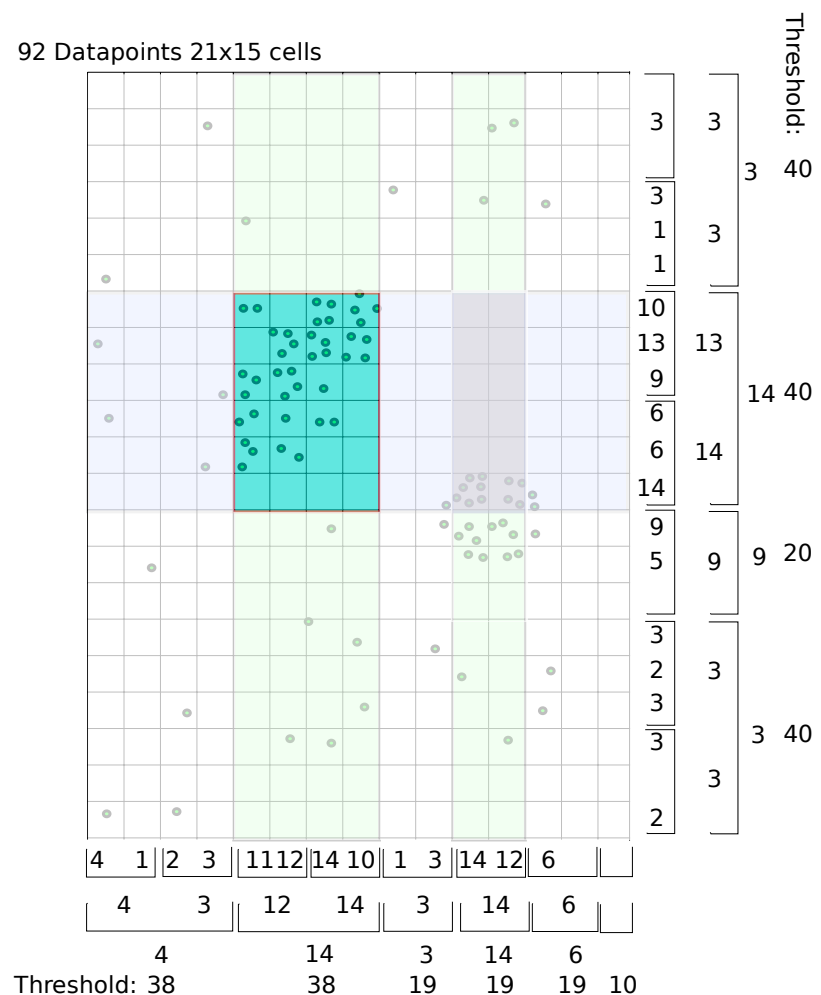

Fig. 5: An illustration of MAFIA using a simple two-dimensional example with $\alpha=1.5$. The light green columns and the light blue row are one-dimensional dense units. The areas where they cross are twodimensional candidates for dense units, but only the darker cyan area is dense. It contains more particles than required by the thresholds of its one-dimensional components.

calculation is done on a single node. But the scaling of the energy calculation when called from Fortran is better than the scaling of the same function called from Python. This may be due to the interplay between mpi4py and JuRoPA's MPI installation and needs further investigation.

Vendors are interested in making Python work on their machines. IBM helped us to improve the scaling of the startup time of Python on our Blue Gene/P. This now makes production runs with more than 100000 cores feasible and reduces the extrapolated time to start Python on the entire machine from more than eight hours to less than one hour.

Still, the goal remains to bring the startup time of the Python interpreter on JUGENE down near that of a regular binary program. We will continue to investigate.

\section{References}

[BioPy] Cock PJ, Antao T, Chang JT, Chapman BA, Cox CJ, Dalke A, Friedberg I, Hamelryck T, Kauff F, Wilczynski B, and de Hoon MJ. Biopython: freely available Python tools for computational molecular biology and bioinformatics. Bioinformatics 25 (11), 1422-3 (2009)

[MOD] Sali A. and Blundell T. L. Comparative protein modelling by satisfaction of spatial restraints. J. Mol. Biol. 234, 779-815 (1993)

[PyLab] Hunter J.D. Matplotlib: A 2D Graphics Environment Computing in Science and Engineering, 9 (3), 90-95 (2007)

[PyQt] Qt - A cross platform application and UI framework, http://qt. nokia.com/

[SMMP] Eisenmenger, F., Hansmann, U.H.E., Hayryan, S. \& Hu, C. [SMMP] A modern package for simulation of proteins. Comp. Phys. Comm. 138, 192-212 (2001).
[SMMP3] Meinke, J.H., Mohanty, S., Eisenmenger, F. \& Hansmann, U.H.E. SMMP v. 3.0 - Simulating proteins and protein interactions in Python and Fortran. Comp. Phys. Comm 178, 459--470 (2007).

[MMTK] Hinsen, K The Molecular Modeling Toolkit: A New Approach to Molecular Simulations J. Comp. Chem. 21, 79--85 (2000)

[Top500] Top 500 List June 2010, http://www.top500.org/list/2010/06/100

[PyOn10k] Python on 10K of cores on BG/P, http://groups.google.com/group/ mpi4py/browse_thread/thread/3dc9b1d9eb153eb3

[GPAW] Mortensen, J.J., Hansen, L.B. \& Jacobsen, K.W. Real-space grid implementation of the projector augmented wave method. Phys. Rev. B 71, 035109 (2005).

[PyMOL] The PyMOL Molecular Graphics System, Version 1.2r3pre, Schroedinger, LLC., http://www.pymol.org/

[MC] Metropolis, N., Rosenbluth, A.W., Rosenbluth, M.N., Teller, A.H \& Teller, E. Equation of state calculations by fast computing machines. J. Chem. Phys. 21, 1087 (1953).

[PT] Hukushima, K. \& Nemoto, K. Exchange Monte Carlo Method and Application to Spin Glass Simulations. J. Phys. Soc. Jpn. 65, 1604-1608 (1996).

[MAFIA] Nagesh, H., Goil, S. \& Choudhary, A. Parallel algorithms for clustering high-dimensional large-scale datasets. Data mining for scientific and engineering applications (2001). 